\title{
Contactless Liquid Sensing Technique Using a Quartz Oscillator
}

\author{
Tsubasa Susa, Takeru Watanabe, Masayuki Sohgawa, and Takashi Abe* \\ Niigata University, 8050, Ikarashi 2-no-cho, Nishi-ku, Niigata 950-2181, Japan
}

(Received October 23, 2015; accepted December 16, 2015)

Keywords: contactless sensing, liquid concentration, quartz crystal resonator, oscillator, factory automation

In this paper, a contactless sensor for the measurement of liquid concentration using a quartz oscillator is reported. An out-of-plane electric field distribution from a planar sensing capacitor (SC) is used for the noncontact sensor measurement of liquid concentration on a spacer. This sensor measures the changes in the capacitance of the SC by the changes in the frequency of the quartz oscillator. Because the capacitance is dependent on the relative permittivity and conductivity of the liquid sample on the spacer, it is possible to measure the concentration of the sample. The response is very stable because, in this sensor, the $\mathrm{SC}$ is incorporated into the quartz oscillator. Because the $\mathrm{SC}$ is in contact with the liquid sample instead of the quartz crystal resonator, the sensor has a high quality factor (approximately 30000). Moreover, this sensor can be used with a general integrated circuit (IC) and an electronic component at a low cost. The sensor is used for the noncontact measurements of liquid concentration.

\section{Introduction}

Liquid sensing technology is used in many applications in industrial automation and waste management for reducing environmental pollution. A typical example is the degradation state of cutting oil and/or lubricating oil in a machining factory. The degradation state of oil is important for reducing energy loss caused for tribological reasons. In such a case, contactless measurement is desirable for practical purposes because the measured liquid is in bottles, tanks, or pipelines. Contactless sensing is also important for hygienic applications.

Electromagnetic techniques with contactless and multifunctional sensing are used for the contactless measurement of the concentration of electrolyte solutions. These techniques are a powerful tool for contactless measurements. ${ }^{(1-5)}$ However, the types of sensors used are relatively expensive and have practical problems in industrial applications. To overcome the drawback of production costs, less expensive sensors are required for industrial applications. In previous studies, a contact-type capacitive sensor for the measurement of liquid concentration with a quartz oscillator had been proposed. ${ }^{(6,7)}$ We proposed a new method for multichannel sensing at a time interval by introducing sample solutions to multiple planer capacitors. ${ }^{(8)}$ An out-of-plane electric field of the planar capacitor was used in our previous study, for which the capacitance depends on the distance between the liquid and the capacitor.

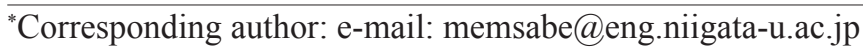


This research focuses on a new contactless sensor for the measurement of liquid concentration with a quartz oscillator. We have determined that the frequency change in a quartz crystal resonator (QCR) is sensitive to the liquid concentration on a spacer because an out-of-plane electric field from a planar capacitor is distributed in a liquid.

\section{Theory and Methods}

\subsection{Principle of the new method}

Figure 1 shows the proposed contactless measurement method for liquid concentration. A planar capacitor is connected in series with a QCR. The QCR was fabricated by patterning a Au electrode on both sides of a crystal purchased from Seiko EG\&G of Japan. The thickness of the crystal was approximately $100 \mu \mathrm{m}$. The electrode size was $2.6 \mathrm{~mm}$. An out-of-plane electric field distribution from planar sensing capacitor (SC) was used for the contactless sensor measurement of a liquid concentration. The relationship between the capacitance change of the $\mathrm{SC}\left(\Delta C_{\mathrm{L}}\right)$ and the frequency change $(\Delta f)$ can be given as

$$
\Delta f=f\left(C_{\mathrm{L} 2}\right)-f\left(C_{\mathrm{L} 1}\right)=-\frac{\Delta C_{\mathrm{L}} C_{1}}{2\left(C_{0}+C_{\mathrm{L} 1}\right)\left(C_{0}+C_{\mathrm{L} 2}\right)} \times \frac{1}{2 \pi \sqrt{L C_{1}}}
$$

where $C_{\mathrm{L} 2}$ is the capacitance after placing a liquid sample on the $\mathrm{SC}, C_{\mathrm{L} 1}$ is the capacitance after placing a reference liquid on a spacer. $L$ is the reactance, $C_{0}$ is the shunt capacitance, and $C_{1}$ is the capacitance of the QCR. The concentration of the liquid on the SC is evaluated from the frequency changes because the capacitance depends on the relative permittivity and conductivity of the liquid sample on the spacer.

The relative permittivity of the liquid depends on the ions and ion pairs in the liquid.(9) Therefore, the conductivity changes are measured by the capacitive change in this method. The absolute value of the frequency depends on the sensor packaging and oscillation circuit. Therefore, the frequency response $(\Delta F)$ for the liquid sample is normalized by the frequency response for distilled water $\left(\Delta F_{\mathrm{w}}\right)$ at a spacer thickness of $1 \mathrm{~mm}$. The normalized frequency response is given as

(a)
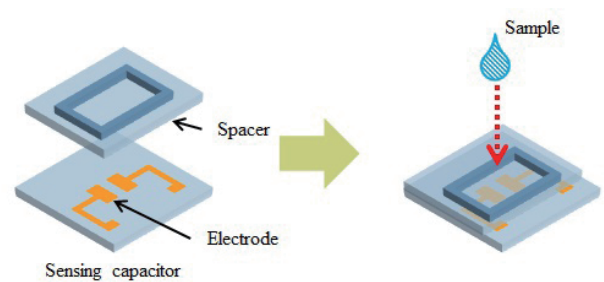

(b)

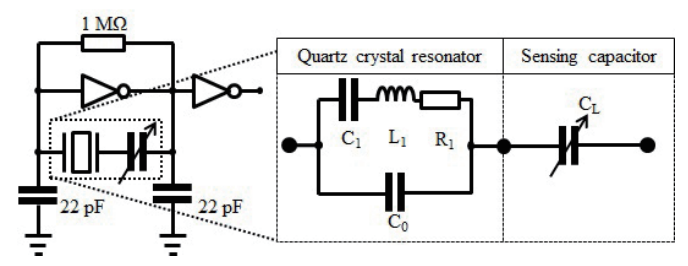

Fig. 1. (Color online) Proposed contactless measurement method for the liquid concentration: (a) a schematic illustration and (b) an equivalent circuit model. 


$$
\Delta F_{\mathrm{n}}=\Delta F / \Delta F_{\mathrm{w}}
$$

In this study, the normalized frequency response was plotted against the concentration of the liquid. Incidentally, the resonant frequency decreased after putting a liquid sample on a spacer as expected [Eq. (1)].

\subsection{Fabricated sensor and its evaluation}

Figure 2 shows an example of a fabricated sensor. The oscillating frequency of the sensor was approximately 16.2 MHz. The capacitors were composed of a pair of Au electrodes (width $=1.3$ $\mathrm{mm}$, length $=2.24 \mathrm{~mm}$, separation $=0.74 \mathrm{~mm}$ ). The substrate used in the capacitor was a glass plate (borosilicate glass). Spacers of different thicknesses $(0.1-6.0 \mathrm{~mm})$ were used to evaluate the frequency response of the sensor against each thickness of the spacer that is modeled as a tank wall. The samples used in this study were prepared by adding ethanol or $\mathrm{NaCl}$ to distilled water. The distilled water is the solution with zero concentration. The capacitance changes were evaluated using an LCR meter (LCR HiTESTER 3535, Hioki, Japan). The conductivity of the $\mathrm{NaCl}$ solution was evaluated with a conductivity meter (Twin-Cond., HORIBA, Japan).

To demonstrate the practical usefulness of the method, the concentration of a water-soluble cutting and grinding fluid was measured. Commercially available fluids (NC-11, SX-542Y, Taiyu Corporation, Japan) were used, and these fluids were diluted 10-30-fold with distilled water. All experiments were conducted at room temperature. Error bars from at least three separate experiments are indicated by the symbols. The detection limits were given by multiplying the sensitivity by three times the standard deviation of the mean frequency variation during $1 \mathrm{~min}$.

\section{Results and Discussion}

\subsection{Evaluation of basic sensor performance}

The sensor was tested in an ethanol/water mixture or $\mathrm{NaCl}$ aqueous solution to evaluate its responses to the relative permittivity and conductivity of the liquid sample.

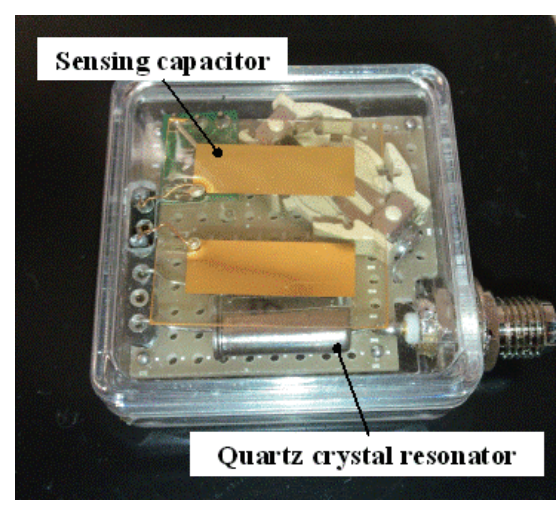

Fig. 2 (Color online) Example of the fabricated device. 
Figure 3 shows the dependence of the normalized frequency change [Eq. (2)] on the ethanol concentration for a spacer thickness of $1 \mathrm{~mm}$. The frequency change $\left(\Delta F_{\mathrm{n}}\right)$ decreases monotonically with increasing ethanol concentration. As suggested by Eq. 1, the sensor frequency response depends on the capacitance change; in fact, the frequency response corresponds to the capacitance change.

Figure 4 shows the dependence of the frequency change on $\mathrm{NaCl}$ concentration. The value of $\Delta F_{\mathrm{n}}$ increases with increasing $\mathrm{NaCl}$ concentration.

The effect of the thickness of the spacer that is modeled as a tank wall on the frequency response was evaluated, because the tank (or pipe) wall thickness suitable for the evaluation depends on each user. Figure 5 shows the relationship between the spacer thickness and the normalized frequency response of the sensor to $\mathrm{NaCl}$ or ethanol concentration. The detection limits are shown in Fig. 6. The detection limits can be improved with thinner spacers. In the case of ethanol, the detection limit for a spacer thickness of $5 \mathrm{~mm}$ is less than $1 \mathrm{wt} \%$. This detection limit can be improved by optimizating the size of the QCR because the frequency response depends on the capacitance of the QCR. ${ }^{(10)}$

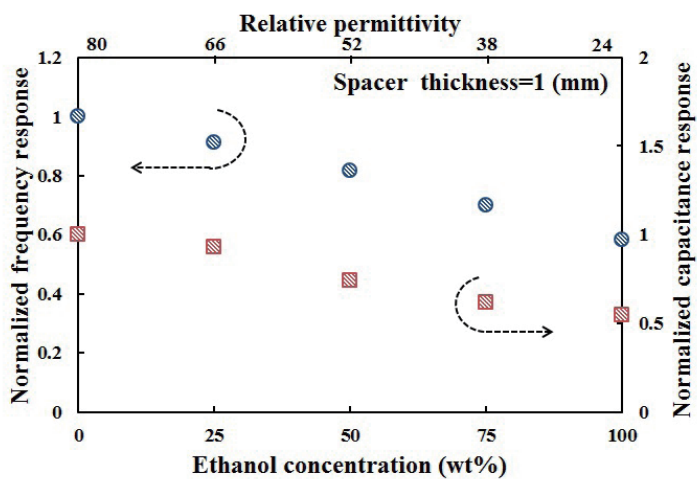

Fig. 3. (Color online) Dependence of the normalized frequency response on the ethanol concentration.

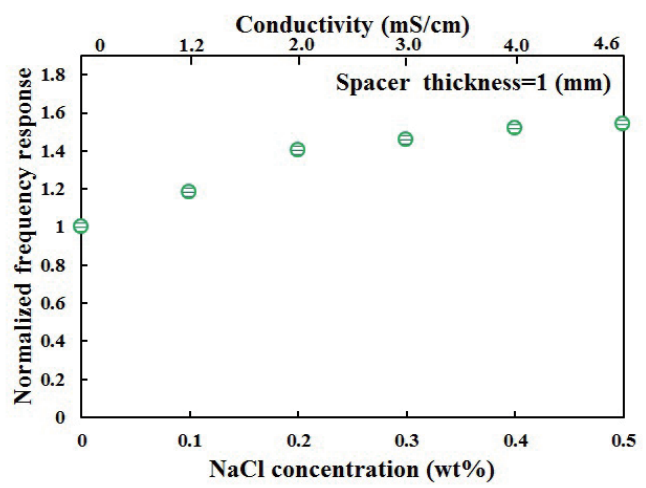

Fig. 4. (Color online) Dependence of the normalized frequency response on the $\mathrm{NaCl}$ concentration.



Fig. 5. (Color online) Relationship between the spacer thickness and the normalized frequency response of $\mathrm{NaCl}$ or ethanol concentration. 


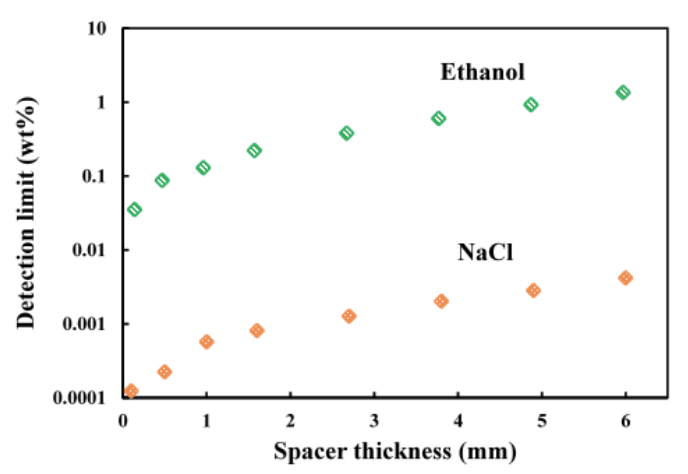

Fig. 6. (Color online) Relationship between the spacer thickness and the detection limit of $\mathrm{NaCl}$ or ethanol concentration.

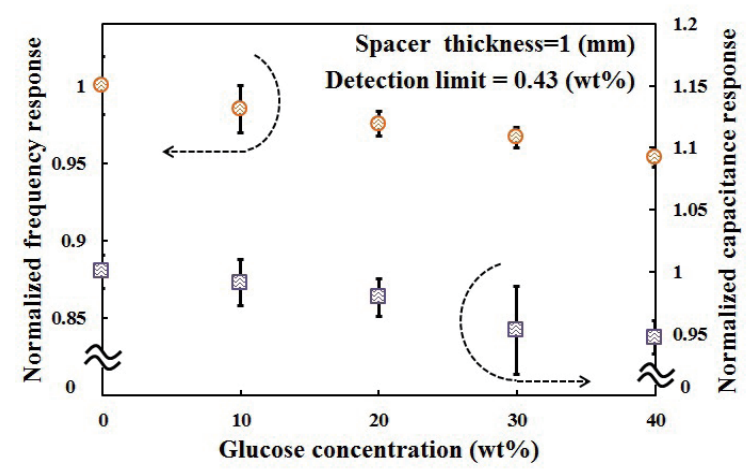

Fig. 7. (Color online) Relationship between the normalized frequency response and the concentration of glucose.

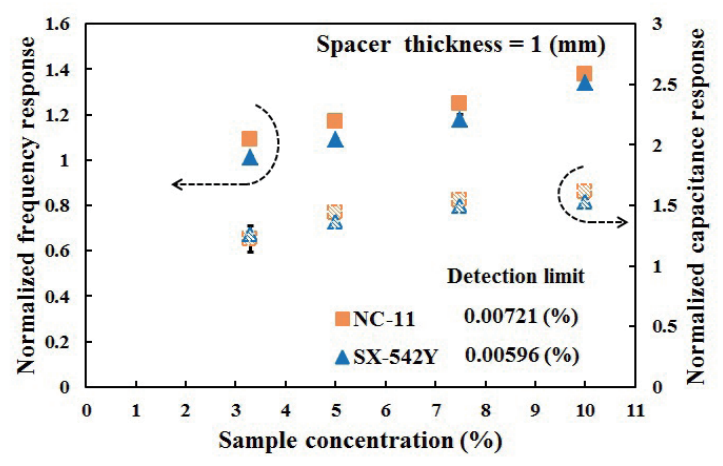

Fig. 8. (Color online) Relationship between the normalized frequency response and the concentration of the grinding fluid sample.

The results in Fig. 7 suggest that the frequency response depends on the glucose concentration. The proposed sensor can measure the glucose concentration because the capacitance changes with the variation in the concentration. The relative permittivities of glucose and distilled water are approximately 3 and 80 at room temperature, respectively. Thus, the relative permittivity of the aqueous solution of glucose depends primarily on the concentration of glucose.

\subsection{Demonstration showing the practical usefulness}

Figure 8 shows the relationship between the normalized frequency change and concentration of the grinding fluid samples for a spacer thickness of $1 \mathrm{~mm}$. The value of $\Delta F_{\mathrm{n}}$ increases with increasing fluid sample concentration. The frequency response corresponds to the capacitance change. An ionized surfactant is used in this fluid sample. The detection limit is less than 0.01 $w t \%$. On the other hand, the required detection limit for the industrial application is $3 \mathrm{wt} \%$. Although these measurements were taken in a laboratory setup, the sensor is expected to be robust in an industrial environment. Comparing the measured data with the database that is created from solutions of known concentration, the proposed contactless sensor is expected to obtain an accurate measurement of liquid concentration. 


\section{Conclusions}

In this paper, a new contactless sensing method for the accurate measurement of liquid concentration is proposed for industrial applications. The proposed sensing method is useful for accurately estimating the concentration of an aqueous solution that contains a single chemical species.

\section{Acknowledgements}

This work was supported in part by the Japan Science and Technology Agency and NAGAI NS Promotion Foundation for Science of Perception.

\section{References}

1 T. Tohyama and H. Yamashita: J. Instrum. Technol. 3 (1957) 146.

2 H. Wakamatsu: Hewlett-Packard Journal 48 (1997) 37.

3 A. Bakar, Md. Ismail, and K. Shida: Sens. Actuators, A 69 (1998) 152.

4 T. Kume, T. Nagano, T. Watanabe, and T. Mitsuno: Trans. JSIDRE 227 (2003) 105.

5 A. Tura, S. Sbrignadello, D. Cianciavicchia, G. Pacini, and P. Ravazzani: Sensors 10 (2010) 5346.

6 S. Ishii, K.Emura, and T Abe: IEEJ Trans. Sens. Micromachines 133 (2013) 96.

7 S. Ishii, K. Emura, and T. Abe: Jpn. J. Appl. Phys. 52 (2013) 127101.

8 S. Toyama and T. Abe: IEEE Sens. J. 14 (2014) 135.

9 T. Sato and R. Buchner: J. Phys. Chem. A 108 (2004) 5007.

10 T. Watanabe, S. Toyama, M. Sohgawa, and T. Abe: IEEJ Trans. Sens. Micromachines 134 (2013) 224. 